\title{
Impact of drifts in edge plasma of small size divertor tokamak
}

\author{
Amr H. Bekheit \\ Plasma \& Nuclear Fusion Department, Nuclear Research Centre, Atomic Energy Authority, Cairo, Egypt; amrbekheitga@yahoo.com
}

Received 29 September 2011; revised 30 October 2011; accepted 12 November 2011

\begin{abstract}
The effect of poloidal $E \times B$ and diamagnetic drifts in edge plasma of Small Size Divertor (SSD) Tokamak is studied with two-dimensional B2SOLPS-0.5.2D fluid transport code. The simulation results show the following: 1) For normal toroidal magnetic field, the increasing of core plasma density leads to large divertor asymmetries due to poloidal $E \times B$ and diamagnetic drifts; 2) Switching on the $E \times B$ and diamagnetic drifts leads to large change in poloidal distribution of radial electric field and induced counter-clockwise circulation (flow) around the $x$-point; 3) Switching on the $E \times B$ and diamagnetic drifts leads to the structure of poloidal distribution of radial electric field is nonmonotonic which responsible for negative spikes; 4) Switching on the $E \times B$ and diamagnetic drifts in vicinity of separatrix leads to the structure of poloidal distribution of radial electric field that has viscous layer; 5) Switching on the $E \times B$ and diamagnetic drifts results in torque generation. This torque spins up the toroidal rotation; 6) The $E \times B$ drift velocity depends on the plasma temperature heating and doesn't depend on plasma density.
\end{abstract}

Keywords: $E \times B$ Drift; Diamagnetic Drifts; B2SOLPS0.5.2D Fluid Transport Code

\section{INTRODUCTION}

The divertor asymmetry has been observed experimentally in several tokamaks with a single null divertor configuration [1-5]. Moreover, the degree of in/out divertor asymmetries depends on the line averaged density $[1,2,5]$. The outer/inner divertor energy asymmetry increases with the heating power from the core plasma for normal toroidal field and decreases with reversed toroidal field [3,5]. Classical particle drifts from $E \times B$ and $\nabla B$ (including curvature) drifts are believed to be important for understanding tokamak edge/scrape off layer (SOL) transport even in the presence of turbulent transport. For example, the asymmetry of the plasma density and temperature in front of the inner and outer divertor plates changes with the sign of the toroidal magnetic field, $B_{T}[1,6]$, and the power threshold for the L-H confinement transition often depends on the direction of $B_{T}[1,6]$. The fluid transport equations of Braginskii [7] are widely used for simulations and describes the drifts of edge tokamak plasma in transport codes such as UEDGE [8,9], EDGE2D [10] and TECXY [11]. In this paper, we focus on a study of the effects of the drifts using the 2-D SOLPS0.5.2D transport code [12,13], including the calculation of $E_{r}$ on both sides of separatrix. This paper demonstrated with core density increasing, the divertor asymmetries due to poloidal $E \times B$ and diamagnetic drifts is large for normal toroidal magnetic field and switching $E \times B$ and diamagnetic drifts leads to large change in poloidal distribution of radial electric field and induced counter-clockwise circulation (flow) around the $\mathrm{x}$-point.

\section{THE MAIN RESULTS OF SIMULATION}

1) The simulations were performed for the parameters of small size divertor tokamak. The small size divertor tokamak is low toroidal magnetic field $\left(B_{T}=1.7 \mathrm{~T}\right), r / a=$ $0.3 \mathrm{~m} / 0.1 \mathrm{~m}$ tokamak. Plasma currents up $50 \mathrm{kA}$ have been achieved. The plasma density and ion temperature in the midplane are: $n=n_{i}=n_{e}=4 \times 10^{19} \mathrm{~m}^{-3}$ and temperature heating $T^{\text {heating }}=0.87 \mathrm{keV}$. The anomalous values of diffusion and heat conductivity coefficients were chosen as follow: $D=0.5 \mathrm{~m}^{2} \cdot \mathrm{s}^{-1}, \chi_{e, i}=0.7 \mathrm{~m}^{2} \cdot \mathrm{s}^{-1}$. The perpendicular viscosity was taken in the form $\eta=n m_{i} D$. The main results of simulation are: The poloidal profiles of plasma density in edge plasma of small size divertor tokamak with drifts on/off are shown in Figures 1 and 2. In case of higher core plasma density, we can see that the poloidal drifts ( $E \times B$ and diamagnetic drifts) clearly strong influences the plasma density in edge plasma of this tokamak. Figure 1 shows that, in the vicinity of the outer target plate ratio of density with and without drifts is 2.73 for normal direction of toroidal magnetic field (i.e. $\nabla B$ is directed towards the $\mathrm{X}$-point), while for inner target plate 


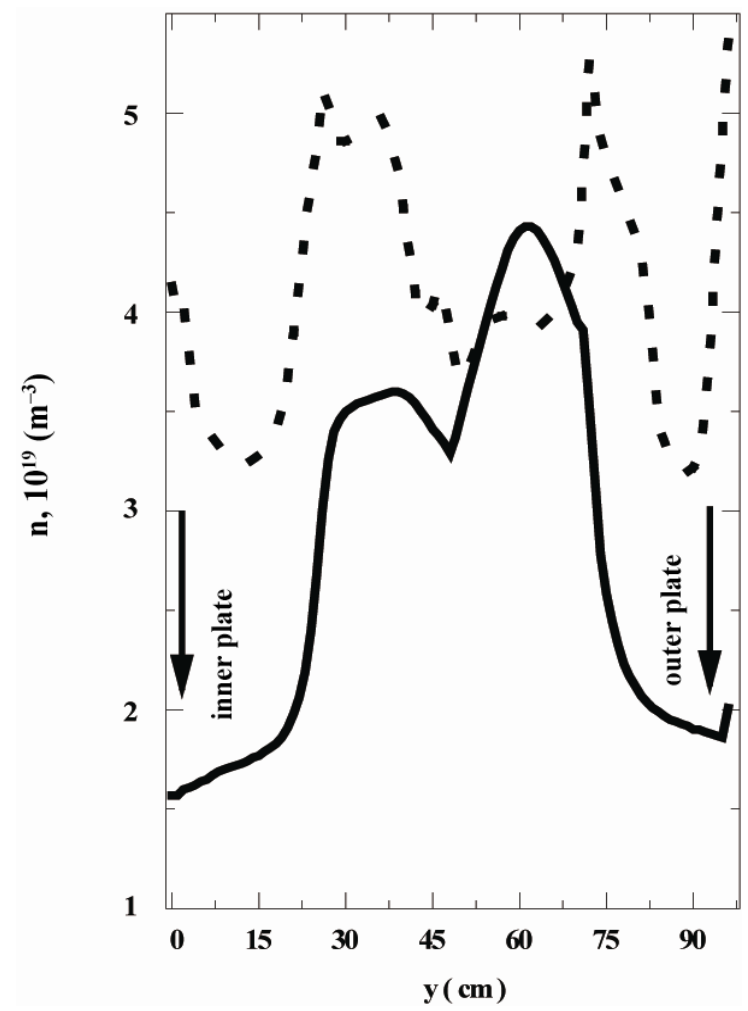

Figure 1. The poloidal distribution of plasma density in edge plasma of small size divertor tokamak. The solid line is density without drifts, but dotted line is density with drifts.

this ratio is equal to 2.63. Also this result shows that for higher core plasma density case the asymmetry in plasma density can change dramatically with line average density as shown as in Figure 2. Figure 2 show the asymmetry of plasma density is low, for low core plasma density case. This result consistent with the result given by $[1,2,4,5]$. In conclusion the change in core plasma density plays a clear role in asymmetry between the divertor legs in edge plasma of small size divertor tokamak.

2) The second result of simulation provides the poloidal distribution of radial electric field with drifts on/off is show in Figure 3. Figure 3 show that, when the drifts switch on the additional radial electric generated in the SOL in the vicinity. The simulated radial electric field can be explain by using [14] as follow: in case of all drifts switch off the presence of radial electric field hill located at inner and outer sides of X-point induces $E \times B$ drift plasma co- and counter circulation around it. Therefore, switching off drifts did not change the radial electric field and flow patter around the X-point. In case of drifts switch on the presence of radial electric field hill located at inner and outer sides of X-point induces $E \times B$ drift plasma co-circulation around it. Therefore, in the normal direction of toroidal magnetic field the plasma flow from outer to inner divertor target below the $\mathrm{X}$-point and hence enhances the recycling efficiency on the inner side

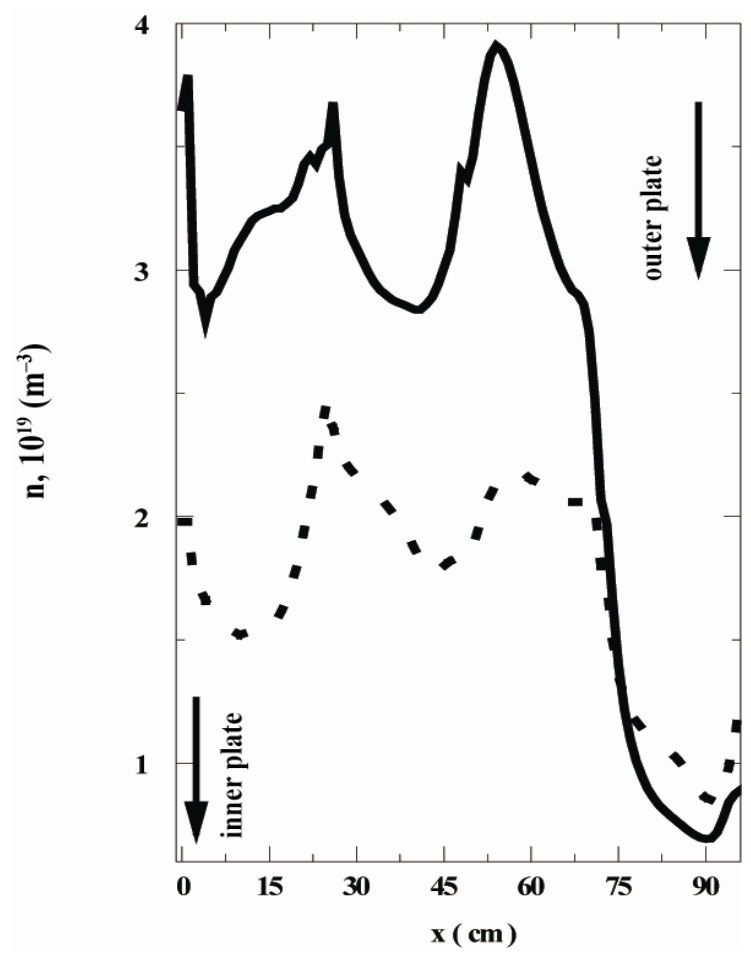

Figure 2. The poloidal distribution of plasma density in edge plasma of small size divertor tokamak with drifts. The solid line is density with higher plasma density core $\left(8 \times 10^{19} \mathrm{~m}^{-3}\right)$, but dotted line is density with drifts with low plasma density core $\left(4 \times 10^{19} \mathrm{~m}^{-3}\right)$.

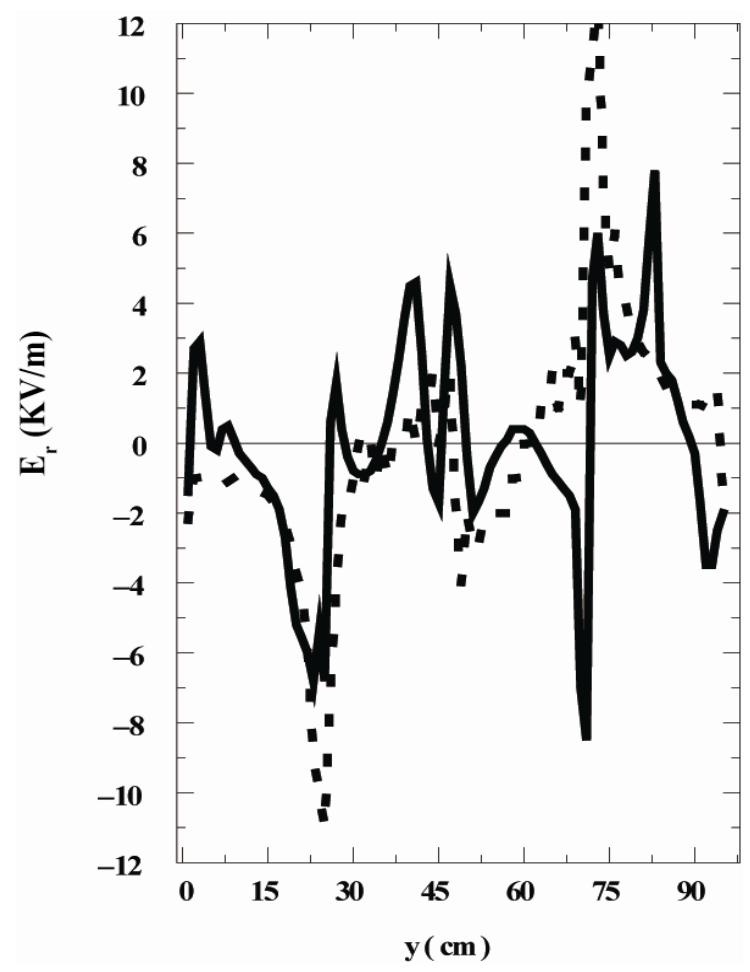

Figure 3. The poloidal distribution of radial electric field with on (solid line)/off (dotted line) drifts in edge plasma of small size divertor tokamak. 
of the X-point. In conclusion in the normal direction of toroidal magnetic the drifts have significant effect on the profile of radial electric field and flow patter around $\mathrm{X}$-point.

3) The third result of simulation shows that, the switch on drifts leads to the polodial distribution of the radial electric field is non-monotonic which is responsible for formation of negative spikes at outer mid-plan as shown in Figure 3. The amplitude of these spikes depends on the difference in potential drop between the plates and equatorial med-plane and controlled by changing SOL parameters [15]. The importance of these strong negative spikes refers to the contribution to turbulence suppression and edge barrier formation.

4) The fourth result of simulation provides that, the structure of the poloidal distribution of radial electric field is very complicated (see Figure 3), and the anomalous transport of parallel momentum play significantly role in the poloidal distribution of radial electric field. Here viscous layer exists which provides the transition from the core to SOL. Inside this layer the parallel viscosity balanced by the radial transport of toroidal momentum. The width of this layer is given by [16]. This result agrees with results given by $[16,17]$.

5) The fifth result of simulation shows that, switch on drifts leads to additional toroidal torque generated in edge plasma of small size divertor tokamak as shown in Figure 4. The mechanism responsible for the toroidal mo-

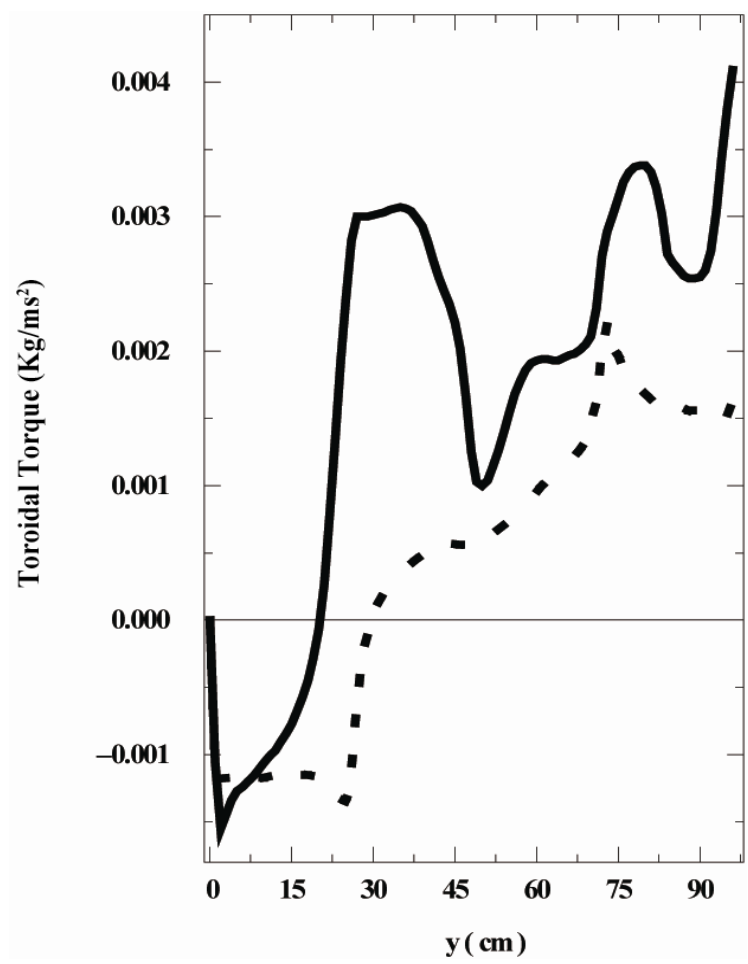

Figure 4. The poloidal distribution of toroidal torque with on (solid line)/off (dotted line) drifts in edge plasma of small size divertor tokamak. mentum torque is illustrated in Figure 4. In the upper part of tours the drifts transport positive toroidal velocity outward (see Figure 5) creating positive torque (see Figure 4). This positive torque is balance mainly by the radial transport of toroidal momentum due to anomalous plasma viscosity and diffusion. One can see that, the plasma viscosity and diffusion play significant role of transport of ion toroidal momentum in edge plasma of small size divertor tokamak.

6) The $E \times B$ drift velocity in edge plasma of small size divertor tokamak in cases of increasing core plasma density and temperature plasma heating are shown in Figures 6 and 7. Figure 6 shows that, for increasing plasma temperature heating the radial electric field inside separatrix is not to a large extent determined by parallel momentum balance equation for electrons [18]. Since inside separatrix the electron temperature increasing with radius the radial electric field here is negative for the normal direction of toroidal magnetic field the $E \times B$ drift velocity is directed from outer to inner plate, but in the SOL the $E \times B$ drift velocity is directed from inner to outer plate. Also Figure 6 show that, the $E \times B$ drift velocity depends on the temperature heating inside separatrix. Figure 7 shows that, the $E \times B$ drift velocity is inde-

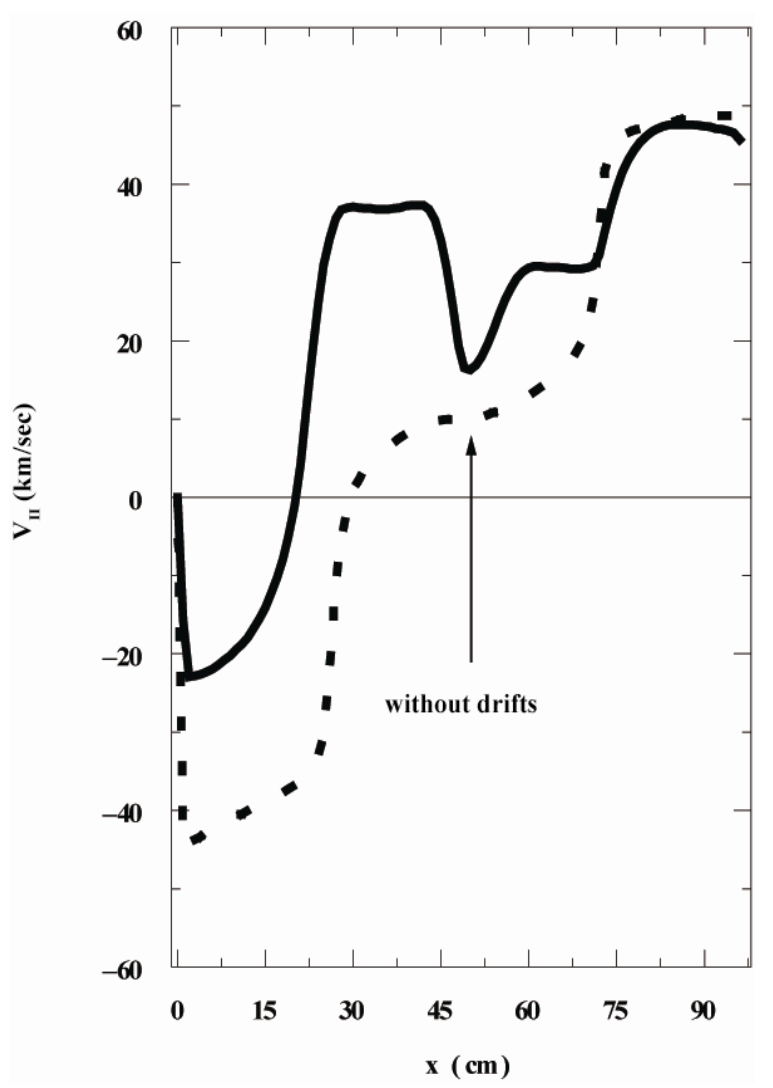

Figure 5. The poloidal distribution of toroidal (parallel) velocity in edge plasma of small size divertor tokamak when drifts switch on (solid line)/off (dotted line). 


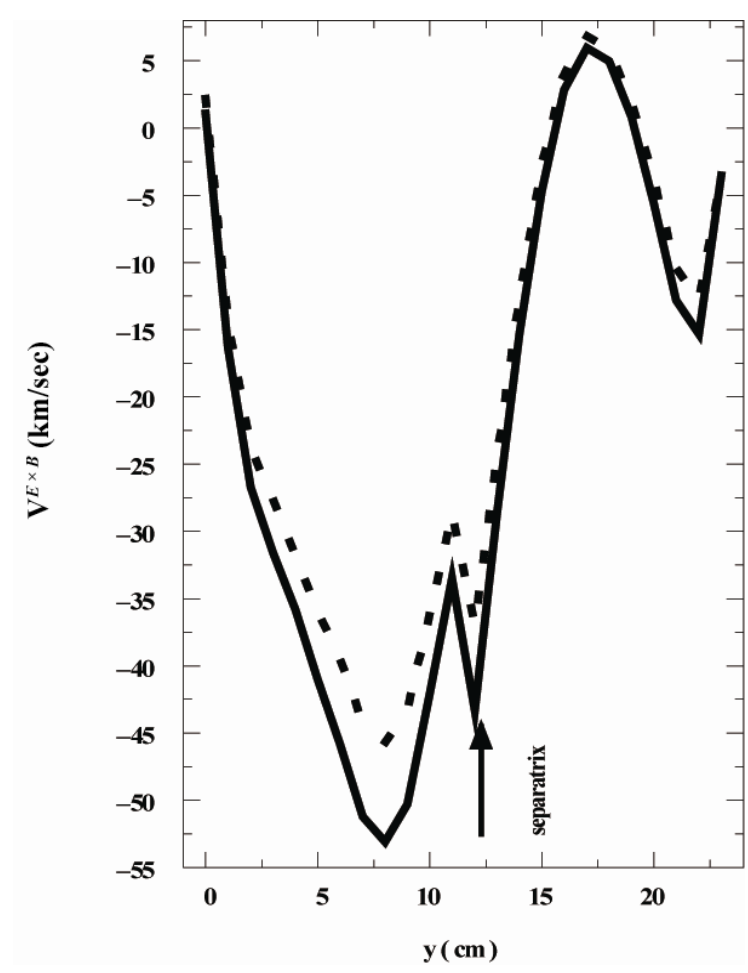

Figure 6. The radial distribution of $E \times B$ drifts velocity at different plasma temperature heating. Dotted line represented $E \times B$ drift velocity at $T^{\text {heating }}=574.4314 \mathrm{eV}$ and solid line represented $E \times B$ drift velocity at $T^{\text {heating }}=$ $643.2814 \mathrm{eV}$.

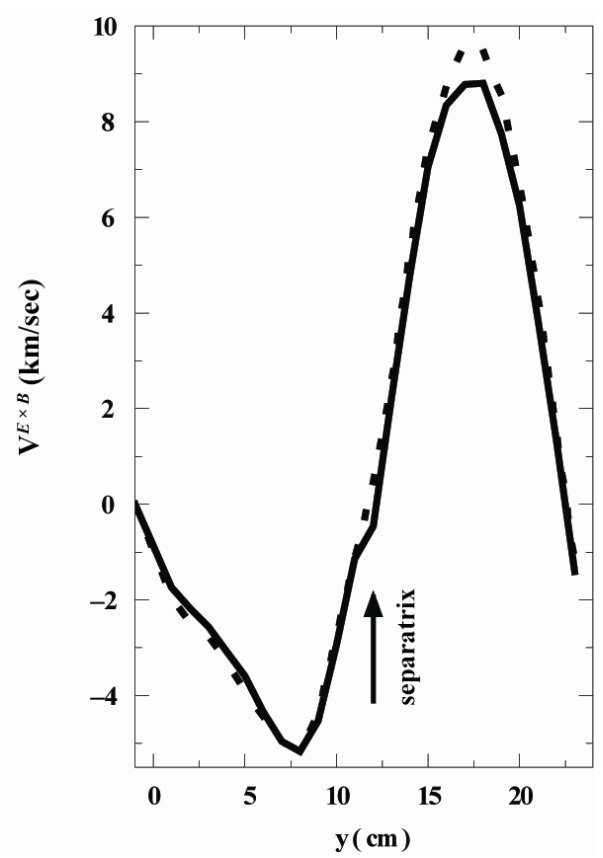

Figure 7. The radial distribution of $E \times B$ drifts velocity at different core plasma density. Dotted line represented $E \times B$ drift velocity at $T^{\text {heating }}=266.61 \mathrm{eV}, n=5 \times 10^{19} \mathrm{~m}^{-3}$ and solid line represented $E \times B$ drift velocity at $T^{\text {heating }}$ $=266.61 \mathrm{eV}, n=4 \times 10^{19} \mathrm{~m}^{-3}$. pendent on core plasma density.

\section{CONCLUSIONS}

The simulation provides the following result:

1) With core density increasing, the divertor asymmetries due to poloidal $E \times B$ and diamagnetic drifts is large for normal toroidal magnetic field.

2) Switching on the $E \times B$ and diamagnetic drifts leads to large change in poloidal distribution of radial electric field and induced counter-clockwise circulation (flow) around the x-point.

3) Switching on the $E \times B$ and diamagnetic drifts leads to the structure of poloidal distribution of radial electric field is non-monotonic which responsible for negative spikes.

4) Switching on the $E \times B$ and diamagnetic drifts in vicinity of separatrix leads to the structure of poloidal distribution of radial electric field has viscous layer.

5) Switching on the $E \times B$ and diamagnetic drifts results in torque generation. This torque spins up the toroidal rotation.

6) The $E \times B$ drift velocity depends on the plasma temperature heating and independence on plasma density.

7) Taking into account the radial electric field and drifts effects is significantly effect in understanding the edge plasma of small size divertor tokamak. These effects are including in the famous codes (e.g. UEDGE and SOLPS. 5.2D). The predictive modeling for modern tokamaks should be performed only with the codes containing these.

\section{REFERENCES}

[1] Hutchinson, I.H, LaBombard, B., Goetz, J.A., Lipschultz, B., McCracken, G.M., Snipes, J.A. and Terry, J.L. (1995) The effects of field reversal on the Alcator C-Mod divertor. Plasma Physics and Controlled Fusion, 37, 13891406. doi:10.1088/0741-3335/37/12/004

[2] Tsois, N., Dorn, C., Kyriadakis, G., Markoulaki, M., Pfug, M., Schramm, G. and ASDEX-Upgread team (1999) A fast scaning langmuir probe system for ASDEX-Upgread Divertor. Journal of Nuclear Materials, 266-269, 12301233. doi:10.1016/S0022-3115(98)00569-8

[3] Pitts, R.A, Andrew, P., Bonnin, X., Chankin, A.V, Corre, Y., Corrigan, G., Coster, D., Duran, I., Eich, T. and contributors to the EFDA-JET workprogramme (2005) Edge and divertor physics with reversed toroidal field in JET. Journal of Nuclear Materials, 337-339, 146-153. doi:10.1016/j.jnucmat.2004.10.111

[4] Ou, J., Zhu, S. (2007) Numerical predictions of the poloidal $E \times B$ drift in EAST. Journal of Nuclear Materials, 363, 633-637.

[5] Bekheit, A. H. and Gaber, W.H. (2010) B2SOLPS0.5.2D code simulations of sol flows and drifts effects in the edge plasma of small size divertor tokamak. Journal of Fusion Energy, 29, 261-266. doi:10.1007/s10894-009-9270-6 
[6] Rognlien, T.D., Porter, G.D. and Ryutov, D.D. (1999) Influence of $E \times B$ and $\nabla B$ drift terms in 2-D edge/SOL transport simulations. Journal of Nuclear Materials, 266269, 654-659. doi:10.1016/S0022-3115(98)00835-6

[7] Braginskii, S.I. (1965) Transport processes in plasma. Reviews of Plasma Physics, 1, 205.

[8] Rognlien, T.D. and Ryutov, D.D. (1998) Analysis of classical transport equations for the tokamak edge plasma. Contributions to Plasma Physics, 38, 152-157. doi:10.1002/ctpp.2150380123

[9] Rognlien, T.D., Ryutov, D.D., Mattor, N. and Porter, G.D. (1999) Two-dimensional electric fields and drifts near the magnetic separatrix in divertor tokamaks. Physics of Plasmas, 6, 1851. doi:10.1063/1.873488

[10] Radford, G.J., Chankin, A.V, Corrigan, G., Simonini, R., Spence, J. and Taroni, A. (1996) The particle and heat drift fluxes and their implementation into the EDGE2D transport code. Contributions to Plasma Physics, 36, 187-191. doi:10.1002/ctpp.2150360217

[11] Gerhauser, R., Zagórski, R., Gerhauser, H. and Claaßen, H.A. (1998) Numerical simulation of the TEXTOR edge plasma including drifts and impurities. Contributions to Plasma Physics, 38, 61-67. doi:10.1002/ctpp.2150380110

[12] Schneider, R., Rozhansky, V., Voskoboynikov, V. (2001) Simulation of tokamak edge plasma including self-consistent electric fields. Nuclear Fusion, 41, 387. doi:10.1088/0029-5515/41/4/305

[13] Bekheit, A.H. (2008) Simulation of small size divertor tokamak plasma edge including self-consistent electric fields. Journal of Fusion Energy, 27, 338-345. doi:10.1007/s10894-008-9148-z

[14] Rozhansky, V., Voskoboynikov, S., Bonnin, X., Coster, D., Schneider, R. and Reiter, D. (2005) Modelling and consequences of drift effects in the edge plasma of Alcator C-Mod. Nuclear Materials, 337-339, 301-304.

[15] Rozhansky, V., Kaveeva, E., Voskoboynikov, S., Counsell, G., Kirk, A., Meyer, H., Coster, D., Conway, G., Schirmer, J., Schneider, R. and the ASDEX Upgrade Team (2006) Modelling of radial electric field profile for different divertor configurations. Plasma Physics and Controlled Fusion, 48, 1425-1435. doi:10.1088/0741-3335/48/9/011

[16] Rozhansky, V., Kaveeva, E., Voskoboynikov, S., Bonnin, X. and Schneider, R. (2004) Understanding transport barriers through modeling. Nuclear Fusion, 42, 1110. doi:10.1088/0029-5515/42/9/309

[17] Rozhansky, V. (2004) Understanding transport barriers through modeling. Plasma Physics and Controlled Fusion, 46, A1. doi:10.1088/0741-3335/46/5A/001

[18] Rozhansky, V., Rozhansky, V., Kaveeva, E., Voskoboynikov, S., Bonnin, X. and Schneider, R., et al. (2003) Modelling of electric fields in tokamak edge plasma and L-H transition. Nuclear Fusion, 43, 1. 